\title{
Prevalence and Costs of Discharge Diagnoses in Inpatient General Internal Medicine: a Multi-center Cross-sectional Study
}

\author{
Amol A. Verma, MD, MPhil ', , Yishan Guo, MSc' ', Janice L. Kwan, MD, MPH', \\ Lauren Lapointe-Shaw, MD2, Shail Rawal, MD, MPH', , Terence Tang, MD, \\ Adina Weinerman, MD, MHSC ${ }^{2,6}$, and Fahad Razak, MD MSC FRCPC $1,2,7,8$
}

\begin{abstract}
'Li Ka Shing Knowledge Institute, St. Michael's Hospital, Toronto, Ontario, Canada; ${ }^{2}$ Department of Medicine, University of Toronto, Toronto, Canada; ${ }^{3}$ Department of Medicine, Mount Sinai Hospital, Toronto, Ontario, Canada; ${ }^{4}$ Division of General Internal Medicine, University Health Network, Toronto, Canada; Institute for Better Health, Trillium Health Partners, Toronto, Canada; ${ }^{5}$ Sunnybrook Health Sciences Centre, Toronto, Canada; ${ }^{7}$ Harvard Center for Population and Development Studies, Cambridge, USA; ${ }^{8}$ Institute of Health Policy, Management and Evaluation, University of Toronto, Toronto, Canada.
\end{abstract}

\begin{abstract}
BACKGROUND: Understanding the most common and costly conditions treated by inpatient general medical services is important for implementing quality improvement, developing health policy, conducting research, and designing medical education.
\end{abstract}

OBJECTIVE: To determine the prevalence and cost of conditions treated on general internal medicine (GIM) inpatient services.

DESIGN: Retrospective cross-sectional study involving 7 hospital sites in Toronto, Canada.

PARTICIPANTS: All patients discharged between April 1, 2010 and March 31, 2015 who were admitted to or discharged from an inpatient GIM service.

MAIN MEASURES: Hospital administrative data were used to identify diagnoses and costs associated with admissions. The primary discharge diagnosis was identified for each admission and categorized into clinically relevant and mutually exclusive categories using the Clinical Classifications Software (CCS) tool.

KEY RESULTS: Among 148,442 admissions, the most common primary discharge diagnoses were heart failure $(5.1 \%)$, pneumonia $(5.0 \%)$, urinary tract infection (4.6\%), chronic obstructive pulmonary disease $(4.5 \%)$, and stroke (4.4\%). The prevalence of the 20 most common conditions was significantly correlated across hospitals (correlation coefficients ranging from 0.55 to $0.95, p \leq 0.01$ for all comparisons). No single condition represented more than $5.1 \%$ of all admissions or more than $7.9 \%$ of admissions at any hospital site. The costliest conditions were stroke (median cost \$7122, interquartile range 5587-12,354, total cost $\$ 94,199,422$, representing $6.0 \%$ of all costs) and the group of delirium, dementia, and cognitive disorders (median cost \$12,831, IQR 9539-17,509, total cost

Prior Presentations: Some of the findings included in this manuscript were presented as a poster at the Canadian Frailty Network 2017 National Conference, April 23, 2017 in Toronto, Canada.

Electronic supplementary material The online version of this article (https://doi.org/10.1007/s11606-018-4591-7) contains supplementary material, which is available to authorized users.

Received February 5, 2018

Revised June 6, 2018

Accepted July 12, 2018

Published online July 27, 2018
$\$ 77,372,541$, representing $4.9 \%$ of all costs). The 10 most common conditions accounted for only $36.2 \%$ of hospitalizations and $36.8 \%$ of total costs. The remaining hospitalizations included 223 different CCS conditions.

CONCLUSIONS: GIM services care for a markedly heterogeneous population but the most common conditions were similar across 7 hospitals. The diversity of conditions cared for in GIM may be challenging for healthcare delivery and quality improvement. Initiatives that cut across individual diseases to address processes of care, patient experience, and functional outcomes may be more relevant to a greater proportion of the GIM population than disease-specific efforts.

KEY WORDS: internal medicine; diagnoses; hospitalist; health services research; cost.

J Gen Intern Med 33(11):1899-904 DOI: $10.1007 / \mathrm{s} 11606-018-4591-7$

(๑) Society of General Internal Medicine 2018

\section{INTRODUCTION}

Understanding the conditions commonly treated by inpatient services is important for healthcare delivery, quality improvement, health policy, research, and education. Innovations to improve healthcare delivery, such as value-based purchasing ${ }^{1}$ and bundled payment reforms, ${ }^{2,3}$ often focus on specific medical conditions. Inpatient subspecialty or surgical services can be optimized for the care of select conditions, as occurs in coronary care or stroke units. However, an increasing number of patients are cared for on general internal medicine (GIM) or hospitalist wards, ${ }^{4,5}$ where care is often provided to patients with a wide variety of conditions. At 7 teaching hospital sites in Toronto, Canada, GIM admissions accounted for 39\% of emergency department admissions to hospital and $24 \%$ of hospital bed-days. ${ }^{6}$ The purpose of our study was to determine the prevalence and cost of conditions treated on GIM inpatient services at these hospitals to inform healthcare delivery, quality improvement, and future research. 


\section{METHODS}

\section{Design and Setting}

We conducted a retrospective cross-sectional study involving 7 hospital sites that were participating in the General Medicine Inpatient Initiative Study (GEMINI) ${ }^{6}$ in the Toronto area, Canada. We included all patients discharged between April 1, 2010 and March 31, 2015 who were admitted to or discharged from an inpatient GIM service. Although most patients were both admitted and discharged from the GIM service, we included patients either admitted to or discharged from GIM. This definition permitted the inclusion of patients who were, for example, initially admitted to an intensive care unit but subsequently cared for and discharged from GIM. GIM services were defined as inpatient services in which the most responsible attending physician cared for general medicine patients in a hospitalist model of care. ${ }^{5}$ The GIM services at GEMINI hospitals have previously been described in detail ${ }^{6}$ and include teaching and non-teaching teams that collaborate with allied health professionals and are staffed by internists (93\%) or family physicians (7\%). The GEMINI sites include 5 academic health centers and 2 large community-based teaching hospitals. Inpatient GIM care is predominantly delivered on clinical teaching units at the academic health centers and by non-teaching services at the community hospitals. Access to inpatient subspecialty wards varied across hospitals and changed within hospitals over the study duration. All hospitals had an inpatient cardiology ward, whereas access to nephrology, pulmonology, gastroenterology, hematology, oncology, stroke units, and other services varied. Research ethics board approval was obtained from all participating hospitals.

\section{Data Sources}

For each patient, we used hospital administrative databases to identify the primary discharge diagnosis that was reported to the Canadian Institute for Health Information ${ }^{7}$ using International Statistical Classification of Diseases and Related Health Problems, Tenth Revision, Canada (ICD-10-CA) codes. The primary diagnosis was defined as "the one diagnosis or condition that can be described as being most responsible for the patient's stay in a facility". 7 Because there are more than 69,800 ICD-10 diagnosis codes and multiple ICD-10 codes may refer to similar conditions, diseases were classified using the Clinical Classifications Software (CCS) tool version $2018.1,{ }^{8}$ which aggregates individual diagnoses into $285 \mathrm{mu}-$ tually exclusive categories, which are mostly clinically homogeneous. The CCS tool that is based on ICD-9 codes has been used widely in clinical and health services research ${ }^{9}$ and ICD10 codes have been mapped using the same process and validated using reverse-mapping to compare against the ICD-9 classifications. $^{8}$ There were 297 ICD-10 codes that could not be automatically categorized in our dataset. These were reviewed manually by a physician (AAV) and matched to a CCS category if the descriptions of the ICD-10 codes were felt to be sufficiently similar to a CCS category. For example, the ICD-10 code R07.4 is "chest pain, unspecified" and this was matched to the CCS category 102, "nonspecific chest pain" (Online Appendix Table S1). To assess for potential bias that may have arisen from the manual matching process, the prevalence of the 20 most common conditions was compared using the automated tool with and without manual matching (Online Appendix Table S2).

To estimate the cost of each hospitalization consistently across organizations, we used the resource intensity weight reported for the Canadian Institute for Health Information multiplied by the average cost per weighted case as estimated by Ontario Cost Distribution Methodology. ${ }^{10}$ This method includes costs related to administration, staff, supplies, and equipment in hospital but not physician billing. Cost data were missing for 48 patients $(<0.1 \%)$ who were excluded from the cost analysis.

\section{Statistical Analysis}

We report the prevalence and median, mean, and total cost of all hospitalizations in Canadian dollars for each CCS condition. We focus on median and total costs because hospital costs in this population are highly right-skewed. ${ }^{6}$ To assess the generalizability of our findings across hospital sites, we compared the prevalence of the 20 most common discharge diagnoses at each hospital with the prevalence of those conditions at the other 6 hospitals combined using Pearson correlation coefficients. We also reported the number of the 20 most common conditions overall that were among the top 20 conditions at each site. Analyses were performed using "R" version 3.3.2 (R Foundation).

\section{RESULTS}

There were 148,442 GIM hospital admissions during the study period involving 3642 unique ICD-10 codes for primary discharge diagnosis. The median age was 73 years (interquartile range 57-83) and the median number of coexisting diagnoses at discharge was 6 (IQR 3-8). Nearly all GIM admissions occurred through the emergency department $(N=144,111$, $97.1 \%)$.

A CCS category was assigned for the primary diagnosis in 137,883 admissions (92.9\%) using the automated tool. Manual assignment of unmatched diagnosis codes enabled the categorization of 147,166 admissions $(99.1 \%)$. The remainder were considered as an "uncategorized" group. Manual matching of CCS codes did not substantially change the prevalence of most common conditions, except non-specific chest pain, which matched fewer than $0.1 \%$ of cases in the automated algorithm but with manual matching was found to have a prevalence of $1.7 \%$ and diabetes mellitus complications, which increased by $0.4 \%$ (Online Appendix Table S1 and S2).

The most common discharge diagnoses (Fig. 1) were heart failure $(5.1 \%)$, pneumonia $(5.0 \%)$, urinary tract infection 


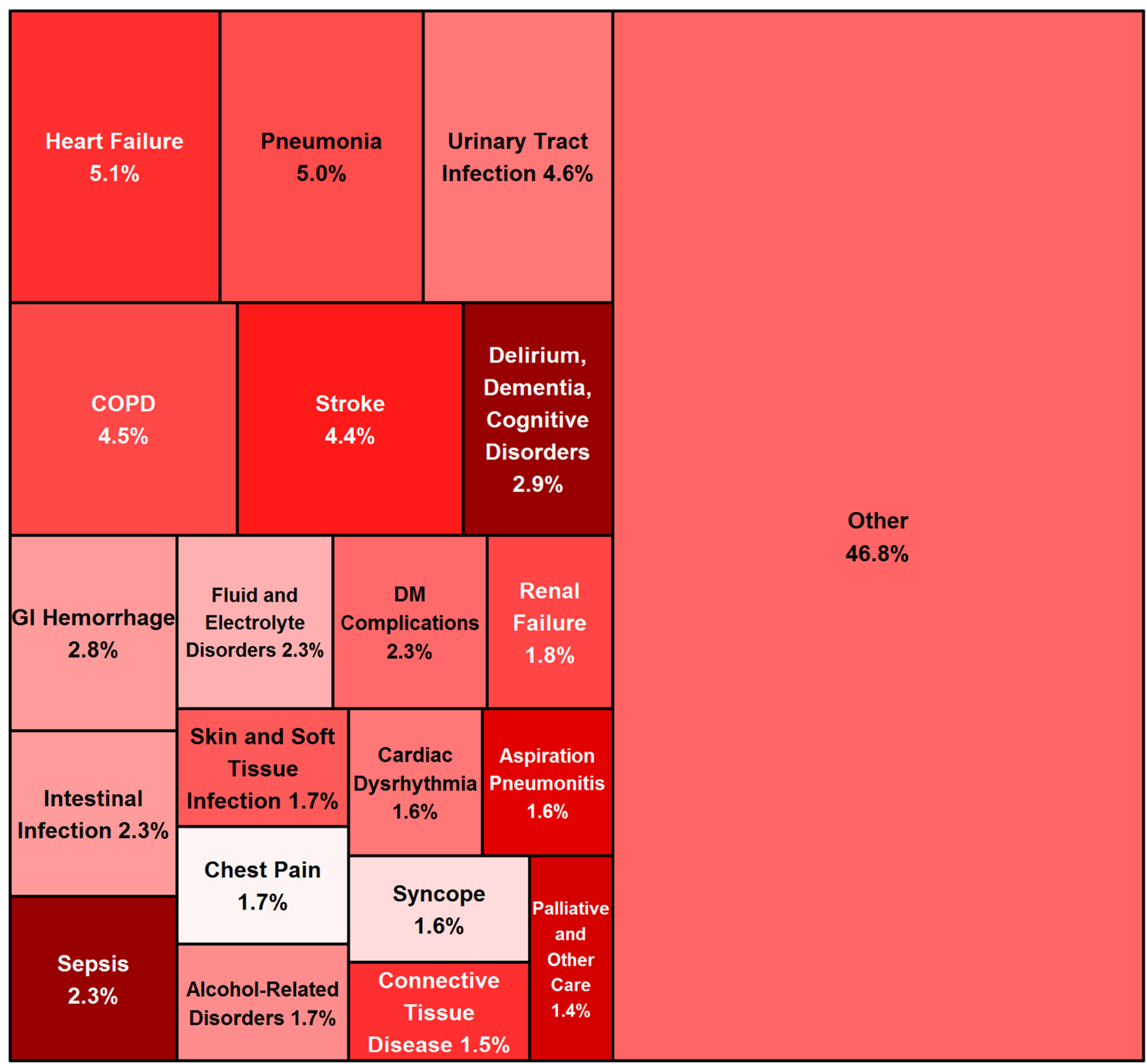

\begin{tabular}{c|ccccc|}
\hline & & & & & \\
\hline 2,000 & 4,000 & 6,000 & 8,000 & 10,000 & 12,000 \\
& Median Cost per Hospitalization & \\
\end{tabular}

Fig. 1 Prevalence and cost of the most common primary discharge diagnoses in GIM. Primary discharge diagnosis was categorized using the CCS tool. Prevalence is denoted by the size of each box, and cost in Canadian dollars is denoted by color. "Other" represents all remaining conditions and is comprised of 213 CCS conditions and one group of "uncategorized" diagnoses (0.9\%). For clarity of presentation, we simplified the description of CCS conditions. COPD: chronic obstructive pulmonary disease, GI; gastrointestinal, DM: diabetes mellitus.

(4.6\%), chronic obstructive pulmonary disease $(4.5 \%)$, and stroke $(4.4 \%)$. No single condition represented more than $5.1 \%$ of all admissions or more than $7.9 \%$ of admissions at any hospital site (Online Appendix Table S3). The conditions with the highest median cost (Fig. 1) were the group of delirium, dementia, and cognitive disorders (median, \$12,831, IQR 9539-17,509; total, \$77,372,541, representing $4.9 \%$ of all costs) and sepsis (median, \$10,025, IQR 606618,954 ; total, $\$ 71,178,460$, representing $4.5 \%$ of all costs).
The condition with the largest total cost was stroke (median, \$7122, IQR 5587-12,354; total, \$94,199,422, representing $6.0 \%$ of all costs, Table 1$)$. The 10 most common conditions accounted for only $36.2 \%$ of hospitalizations and $36.8 \%$ of total costs. The remaining hospitalizations included 223 different CCS conditions (Online Appendix Table S3).

The prevalence of the 20 most common CCS conditions was very similar across 6 of the 7 hospitals, with correlations ranging from 0.77 to 0.95 ( $p<0.001$, Table 2$)$. Site $\mathrm{G}$ was less 
Table 1 Costs of Hospital Admissions for Primary Discharge Diagnoses in GIM, Ranked by Total Cost

\begin{tabular}{|c|c|c|c|c|}
\hline Condition & $\begin{array}{l}\text { Cost } \\
\text { median (IQR) }\end{array}$ & $\begin{array}{l}\text { Cost } \\
\text { mean (SD) }\end{array}$ & $\begin{array}{l}\text { Cost } \\
\text { total }\end{array}$ & $\begin{array}{l}\text { Percentage } \\
\text { of total costs }\end{array}$ \\
\hline Stroke & $7122(5587,12,354)$ & $14,368(29,264)$ & $94,199,422$ & 6.0 \\
\hline Delirium, dementia, cognitive disorders & $12,831(9593,17,509)$ & $17,807(20,960)$ & $77,372,541$ & 4.9 \\
\hline Heart failure & $6717(4955,9723)$ & $10,105(13,701)$ & $77,189,184$ & 4.9 \\
\hline Pneumonia & $6009(4350,8658)$ & $10,036(17,716)$ & $74,084,705$ & 4.7 \\
\hline Sepsis & $10,025(6066,18,954)$ & $20,728(40,657)$ & $71,178,460$ & 4.5 \\
\hline Chronic obstructive pulmonary disease & $6148(4459,8741)$ & $9479(14,751)$ & $62,609,639$ & 4.0 \\
\hline Respiratory failure & $21,321(9156,56,373)$ & $57,449(130,108)$ & $56,300,074$ & 3.6 \\
\hline Urinary tract infection & $5071(3923,7205)$ & $7156(9369)$ & $49,303,691$ & 3.1 \\
\hline Aspiration pneumonitis & $8350(6043,13,147)$ & $17,423(62,307)$ & $41,763,796$ & 2.6 \\
\hline Diabetes mellitus complications & $5381(3699,9792)$ & $10,895(22,069)$ & $36,464,760$ & 2.3 \\
\hline
\end{tabular}

Admissions to GIM were categorized by primary discharge diagnosis using the CCS tool. Conditions were ranked by total cost over the study period. For clarity of presentation, we simplified the description of CCS conditions. Costs are presented in Canadian dollars. IQR: interquartile range

similar, but the prevalence of the most common conditions was still significantly correlated with the other sites (correlation $0.55, p=0.01)$. Admissions for acute myocardial infarction $(2.2 \%)$ and coronary atherosclerosis $(2.3 \%)$ were significantly more common at site $\mathrm{G}$ than in the 6 other sites combined $(0.5$ and $0.9 \%$, respectively, $p<0.001$ for both comparisons). At least 15 of the 20 most common conditions overall were among the top 20 conditions at each site (Table 2).

\section{DISCUSSION}

This multi-center cross-sectional study found that the most common discharge diagnoses in GIM admissions were heart failure, pneumonia, urinary tract infection, chronic obstructive pulmonary disease, and stroke. No single condition accounted for more than $5.1 \%$ of GIM admissions, highlighting the striking heterogeneity of this population. The costliest conditions per admission were sepsis and the group of delirium, dementia, and cognitive disorders. Although referral patterns and models of care vary across participating hospitals, ${ }^{6}$ the prevalence of conditions in our analysis was highly correlated across most sites.

Table 2 Correlations Between Hospital Sites in the Prevalence of the 20 Most Common Discharge Diagnoses in GIM

\begin{tabular}{llll}
\hline \hline $\begin{array}{l}\text { Hospital } \\
\text { site }\end{array}$ & \multicolumn{2}{l}{ Prevalence of common conditions } \\
\cline { 2 - 4 } & $\begin{array}{l}\text { Correlation } \\
\text { with } 6 \text { other } \\
\text { sites }\end{array}$ & $p$ value & $\begin{array}{l}\text { Number of 20 most } \\
\text { common diagnoses } \\
\text { overall among the } \\
\text { site's top 20 }\end{array}$ \\
\hline A & 0.85 & $<0.001$ & 17 \\
B & 0.95 & $<0.001$ & 18 \\
C & 0.77 & $<0.001$ & 15 \\
D & 0.79 & $<0.001$ & 17 \\
E & 0.80 & $<0.001$ & 16 \\
F & 0.80 & $<0.001$ & 16 \\
G & 0.55 & 0.01 & 16 \\
\hline
\end{tabular}

To assess the generalizability of our findings across hospital sites, the prevalence of the 20 most common CCS conditions at each hospital was compared to the prevalence of those conditions at the other 6 hospitals combined using Pearson correlation coefficients
The diversity of conditions cared for by GIM inpatient services is unique, even when compared to other generalist inpatient services. The 10 most common conditions accounted for only $36.2 \%$ of hospitalizations in GIM compared with $83.3 \%$ of emergency general surgery hospitalizations ${ }^{11}$ and $72.6 \%$ of emergency intensive care unit admissions. ${ }^{12}$ This poses important challenges for healthcare delivery, quality improvement, performance measurement, education, and research in GIM. Healthcare reforms often focus on individual diseases, such as the episodes of care in the Medicare Bundled Payments for Care Improvement Intitiative. ${ }^{2}$ Disease-specific process and outcome measures are an important part of performance measurement and value-based purchasing initiatives. ${ }^{1}$ In order to achieve system-wide impact, these innovations must be effective in GIM, whose patients represent nearly $40 \%$ of emergency department admissions. ${ }^{6}$ However, disease-specific approaches may neglect many GIM patients. Even the 20 most common conditions account for only half of admissions to GIM. Our findings suggest that GIM care should be guided by broad metrics that can be applied across diseases. Quality improvement in GIM may have greater impact by focusing on common processes such as transitions in care, ${ }^{13}$ medication safety,${ }^{14}$ or functional status, ${ }^{15}$ rather than on individual diseases.

Disease-specific approaches to healthcare delivery, quality improvement, and research will nevertheless remain important. Further, the shift toward competency-based medical education ${ }^{16}$ will require workplace-based assessments to establish competency in the care of common and complex conditions. ${ }^{17}$ To inform these efforts, we provide data about the most burdensome conditions cared for in GIM, both in terms of frequency and cost.

Although many studies are conducted in GIM inpatient populations, ${ }^{18-22}$ very few explicitly evaluate the most common conditions cared for in GIM. A study of 1501 consecutive patients in 18 European countries over a 3-month period found that the most common conditions on internal medicine hospital wards were pneumonia $(8.2 \%)$, heart failure $(6.3 \%)$, atrial fibrillation/flutter (3.1\%), chronic obstructive pulmonary disease $(3.1 \%)$, and urinary tract infection $(2.7 \%))^{23}$ This study was limited by relatively small sample size and short duration 
(thus being vulnerable to seasonal effects or sampling errors). Our study builds on the existing literature by providing comprehensive capture of a large cohort of GIM admissions at 7 hospitals over a 5-year period and employing the CCS tool to categorize admissions into clinically relevant conditions. A strength of our work is the ability to use hospital-specific data to clearly identify the patients that were cared for by GIM services as opposed to subspecialty services, which may not be possible with large administrative or claims-based datasets, particularly when GIM patients are admitted to off-service units. Thus, our findings reflect the true scope of GIM practice at study hospitals. Furthermore, we provide data about the prevalence of all CCS conditions, not only the most common ones, in a supplementary online appendix for readers who require more detailed information.

The first main limitation of our study was identifying common diagnoses using ICD-10 codes, which are numerous, not necessarily mutually exclusive, and assigned to patients for administrative, and not clinical, reasons. However, it is unlikely that the observed heterogeneity of diagnoses in our study is simply an artifact of coding. The CCS tool has been specifically designed to address the problems caused by numerous overlapping ICD codes. Furthermore, our findings are similar to the multi-center European study by Duckitt and colleagues, ${ }^{23}$ which reported clinical, not administrative, diagnoses. Second, CCS categories are sometimes limited in the granularity of clinical information they provide, particularly pertaining to disease severity. For example, the diagnosis "urinary tract infection" may include a spectrum of disorders ranging from cystitis to pyelonephritis. However, reporting clinically meaningful categories from several thousand ICD10 codes is an important strength of the CCS classification and was felt to outweigh this limitation because our aim was to provide an overall summary of the conditions cared for in GIM. Third, our study was conducted in 5 academic health centers and 2 large community hospitals and it is possible that our findings may not be generalizable to smaller non-teaching hospitals. We found very strong correlations in the prevalence of conditions across most sites and moderately strong correlation at 1 site. Moreover, at least 15 of the 20 most common conditions overall were among the top 20 conditions at each site. The consistency of our findings across a range of urban and suburban hospitals with different models of teaching and non-teaching GIM services, and different access to inpatient subspecialty wards, strengthens our confidence in the generalizability of our findings. Further, in addition to similarity to the study by Duckitt and colleagues, ${ }^{23}$ the GEMINI study cohort was previously reported ${ }^{6}$ to be broadly similar to other GIM populations in Canada, ${ }^{18,19}$ Europe, ${ }^{20,24}$ and the USA. ${ }^{22}$

GIM services represent a large proportion of inpatient care and treat patients with a diversity of diseases. The heterogeneity of conditions cared for in GIM poses important challenges for healthcare delivery. Initiatives that cut across individual diseases and address processes of care, ${ }^{18}$ patient experience, ${ }^{25}$ and functional outcomes ${ }^{15}$ will be more likely to improve care for all GIM patients.

Corresponding Author: Amol A. Verma, MD, MPhil; Li Ka Shing Knowledge Institute, St. Michael's Hospital, Room 714-3, 2 Queen St. East, Toronto, Ontario M5C 3G7, Canada (e-mail: amol.verma@mail. utoronto.ca).

Funders GEMINI was supported by grants from Green Shield Canada Foundation and the University of Toronto Division of General Internal Medicine.

\section{Compliance with ethical standards:}

Research ethics board approval was obtained from all participating hospitals.

Prior presentations: Some of the findings included in this manuscript were presented as a poster at the Canadian Frailty Network 2017 National Conference, April 23, 2017 in Toronto, Canada..

Conflict of interest: The authors declare that they do not have a conflict of interest.

\section{REFERENCES}

1. VanLare JM, Conway PH. Value-based Purchasing - National Programs to Move from Volume to Value. N Engl J Med. 2012;367(4):292-295. doi:https://doi.org/10.1056/NEJMp1204939.

2. MJ Press, Rajkumar R, PH Conway. Medicare's new bundled payments: Design, strategy, and evolution. JAMA. 2016;315(2):131-132. doi: https://doi.org/10.1001/jama.2015.18161.

3. Verma A, Bhatia S. A policy framework for health systems to promote triple aim innovation. Healthc Pap. 2016;15(3):9-23.

4. Soong C, Fan E, Howell EE, et al. Characteristics of hospitalists and hospitalist programs in the united states and canada. $J$ Clin Outcomes Manag. 2009;16(2):69-74.

5. Wachter RM, Goldman L. Zero to 50,000 - the 20th Anniversary of the Hospitalist. N Engl J Med. 2016;375(11):1009-1011. doi:https://doi.org/ 10.1056/NEJMp1607958.

6. Verma AA, Guo Y, Kwan JL, et al. Patient characteristics, resource use and outcomes associated with general internal medicine hospital care: the General Medicine Inpatient Initiative (GEMINI) retrospective cohort study. CMAJ Open. 2017;5(4):E842-E849. doi:https://doi.org/10.9778/ cmajo.20170097.

7. Canadian Institute for Health Information. DAD Abstracting Manual, 2015-2016 Edition. Ottawa, ON; 2015.

8. Healthcare Cost and Utilization Project (HCUP). Beta Clinical Classifications Software (CCS) for ICD-10-CM/PCS. Agency for Healthcare Research and Quality. https://www.hcup-us.ahrq.gov/toolssoftware/ ccs 10/ccs 10.jsp. Published 2017. Accessed July 9, 2018.

9. Healthcare Cost and Utilization Project (HCUP). Publications Using CCS. Agency for Healthcare Research and Quality. https://www.hcup-us.ahrq gov/toolssoftware/ccs/ccs.jsp\#pubs. Published 2017. Accessed July 9, 2018.

10. Wodchis WP, Bushmeneva K, Nikitovic M, McKillop I. Guidelines on Person-Level Costing Using Administrative Databases in Ontario. Toronto, Ontario; 2013.

11. Ogola GO, Shafi S. Cost of specific emergency general surgery diseases and factors associated with high-cost patients. J Trauma Acute Care Surg. 2016;80(2):265-271. doi:https://doi.org/10.1097/TA. 0000000000000911.

12. Simpson HK, Clancy M, Goldfrad C, Rowan K. Admissions to intensive care units from emergency departments: a descriptive study. Emerg Med J. 2005;22(6):423-428. doi:https://doi.org/10.1136/emj.2003.005124.

13. Snow V, Beck D, Budnitz T, et al. Transitions of Care Consensus policy statement: American College of Physicians, Society of General Internal Medicine, Society of Hospital Medicine, American Geriatrics Society, American College Of Emergency Physicians, and Society for Academic 
Emergency M. J Hosp Med. 2009;4(6):364-370. doi:https://doi.org/10. 1002/jhm.510.

14. Ravn-Nielsen L, Duckert M, Lund M, et al. Effect of an in-hospital multifaceted clinical pharmacist intervention on the risk of readmission: a randomized clinical trial. JAMA Intern Med. 2018. doi: https://doi.org/ $10.1001 /$ jamainternmed.2017.8274

15. Liu B, Moore JE, Almaawiy U, et al. Outcomes of Mobilisation of Vulnerable Elders in Ontario (MOVE ON): a multisite interrupted time series evaluation of an implementation intervention to increase patient mobilisation. Age Ageing. 2018;47(1):112-119. doi:https://doi.org/10. 1093/ageing/afx128.

16. Caverzagie KJ, Nousiainen MT, Ferguson PC, et al. Overarching Challenges to the Implementation of Competency-Based Medical Education. Med Teach. 2017;39(6):588-593. doi:https://doi.org/10.1080/ 0142159X.2017.1315075.

17. Caverzagie KJ, Cooney TG, Hemmer PA, Berkowitz L. The Development of Entrustable Professional Activities for Internal Medicine Residency Training: a Report from the Education Redesign Committee of the Alliance for Academic Internal Medicine. Acad Med. 2015;90(4):479-484. doi:https://doi.org/10.1097/ACM.0000000000000564.

18. McAlister FA, Bakal JA, Majumdar SR, et al. Safely and Effectively Reducing Inpatient Length of Stay: a Controlled Study of the General Internal Medicine Care Transformation Initiative. BMJ Qual Saf. 2014;23(1):446-456. doi:https://doi.org/10.1136/bmjqs-2013-002289.

19. McAlister FA, Youngson E, Bakal JA, Holroyd-Leduc J, Kassam N. Physician Experience and Outcomes Among Patients Admitted to General
Internal Medicine Teaching Wards. Can Med Assoc J. 2015;187(14):10411048. doi:https://doi.org/10.1503/cmaj.150316.

20. Kellett J, Deane B. The Diagnoses and Co-morbidity Encountered in the Hospital Practice of Acute Internal Medicine. Eur J Intern Med. 2007;18(6):467-473. doi:https://doi.org/10.1016/j.ejim.2007.02.019.

21. Buurman BM, Frenkel WJ, Abu-Hanna A, Parlevliet JL, De Rooij SE. Acute and Chronic Diseases as Part of Multimorbidity in Acutely Hospitalized Older Patients. Eur J Intern Med. 2016;27:68-75. doi:https://doi.org/10.1016/j.ejim.2015.09.021.

22. Ong M, Bostrom A, Vidyarthi A, McCulloch C, Auerbach A. House Staff Team Workload and Organization Effects on Patient Outcomes in an Academic General Internal Medicine Inpatient Service. Arch Intern Med. 2007;167(1):47-52. doi: https://doi.org/10.1001/archinte.167.1.47.

23. Duckitt R, Palsson R, Bosanska L, Dagna L, Mine Durusu T, Vardi M. Common Diagnoses in Internal Medicine in Europe 2009: a panEuropean, Multi-Centre Survey. Eur J Intern Med. 2010;21(5):449-452. doi:https://doi.org/10.1016/j.ejim.2010.06.012.

24. Buurman BM, Frenkel WJ, Abu-Hanna A, Parlevliet JL, de Rooij SE. Acute and Chronic Diseases as Part of Multimorbidity in Acutely Hospitalized Older Patients. Eur J Intern Med. 2016;27:68-75. doi: https://doi.org/10.1016/j.ejim.2015.09.021.

25. Davidson KW, Shaffer J, Ye S, et al. Interventions to Improve Hospital Patient Satisfaction with Healthcare Providers and Systems: a Systematic Review. BMJ Qual Saf. 2017;26(7):596-606. doi:https://doi.org/10. 1136/bmjqs-2015-004758. 